\title{
Optimisation of quantitative lung SPECT applied to mild COPD: a software phantom simulation study
}

Pernilla Norberg ${ }^{1,2^{*}}$, Anna Olsson 1,2,3, Gudrun Alm Carlsson ${ }^{1,2}$, Michael Sandborg ${ }^{1,2}$ and Agnetha Gustafsson ${ }^{4}$

\begin{abstract}
Background: The amount of inhomogeneities in a ${ }^{99 \mathrm{~m}} \mathrm{Tc}$ Technegas single-photon emission computed tomography (SPECT) lung image, caused by reduced ventilation in lung regions affected by chronic obstructive pulmonary disease (COPD), is correlated to disease advancement. A quantitative analysis method, the $\mathrm{CV}_{\mathrm{T}}$ method, measuring these inhomogeneities was proposed in earlier work. To detect mild COPD, which is a difficult task, optimised parameter values are needed.

Methods: In this work, the $\mathrm{CV}_{T}$ method was optimised with respect to the parameter values of acquisition, reconstruction and analysis. The ordered subset expectation maximisation (OSEM) algorithm was used for reconstructing the lung SPECT images. As a first step towards clinical application of the $\mathrm{CV}_{T}$ method in detecting mild COPD, this study was based on simulated SPECT images of an advanced anthropomorphic lung software phantom including respiratory and cardiac motion, where the mild COPD lung had an overall ventilation reduction of $5 \%$.
\end{abstract}

Results: The best separation between healthy and mild COPD lung images as determined using the $\mathrm{CV}_{T}$ measure of ventilation inhomogeneity and $125 \mathrm{MBq}{ }^{99 \mathrm{~m}} \mathrm{Tc}$ was obtained using a low-energy high-resolution collimator (LEHR) and a power 6 Butterworth post-filter with a cutoff frequency of 0.6 to $0.7 \mathrm{~cm}^{-1}$. Sixty-four reconstruction updates and a small kernel size should be used when the whole lung is analysed, and for the reduced lung a greater number of updates and a larger kernel size are needed.

Conclusions: A LEHR collimator and $125^{99 \mathrm{~m} T c}$ MBq together with an optimal combination of cutoff frequency, number of updates and kernel size, gave the best result. Suboptimal selections of either cutoff frequency, number of updates and kernel size will reduce the imaging system's ability to detect mild COPD in the lung phantom.

Keywords: SPECT; Quantitative evaluation; Lung diseases; Computer-assisted image analysis; Technegas; Simulation

\section{Background}

Obstructed airways and parenchymal destruction are characteristics of chronic obstructive pulmonary disease (COPD). Varying degrees of abnormalities are typically found in different parts of the COPD lung, and some parts of the lung may even be normal. Therefore, the first

\footnotetext{
* Correspondence: pernilla.norberg@regionostergotland.se

'Medical Radiation Physics, Department of Medical and Health Sciences,

Linköping University, Linköping 581 83, Sweden

${ }^{2}$ Center for Medical Image Science and Visualization (CMIV), Linköping

University, Linköping 581 83, Sweden

Full list of author information is available at the end of the article
}

abnormality to be detected in the early stages of the disease is abnormal ventilation distribution [1]. Since the lung behaves in such an irregular manner, these regional differences in ventilation are preferably studied with the use of single-photon emission computed tomography (SPECT) imaging [2]. Early detection of lung function reduction is important in order to prevent further lung degeneration.

We have previously presented the quantitative $\mathrm{CV}_{\mathrm{T}}$ method $[3,4]$ which measures inhomogeneities in lung SPECT images. The purpose of the $\mathrm{CV}_{\mathrm{T}}$ method is to discriminate between activity distributions in the lungs of 
healthy subjects and subjects with mild COPD. The method has been reported in detail previously. Briefly, the coefficient of variation $(\mathrm{CV}=$ the standard deviation divided by the mean) is calculated for overlapping cubic volumes covering the $3 \mathrm{D}$ reconstructed activity distribution. The $\mathrm{CV}$ values are plotted as a density curve with an area under the curve (AUC) of $100 \%$. A diseased lung is characterised by larger proportion of high $\mathrm{CV}$ values compared to a healthy lung. The proportion of high CV values increases with disease advancement (increased heterogeneity of the activity distribution). A CV threshold value $\left(\mathrm{CV}_{\mathrm{T}}\right)$ has been determined as the most frequently occurring $\mathrm{CV}$ value in the distributions of healthy subjects. Finally, the $\operatorname{AUC}\left(\mathrm{CV}_{\mathrm{T}}\right)$, the area under the density curve (AUC) for $\mathrm{CV}$ values greater than $\mathrm{CV}_{\mathrm{T}}$, is calculated for both healthy subjects and subjects with COPD. The method has been shown to be capable of identifying simulated mild COPD in an anthropomorphic software phantom (sensitivity $=95 \%$, specificity $=90 \%)$ and patients with severe COPD (sensitivity $=100 \%$, specificity $=100 \%$ ). The parameter values used in that study [3] were not optimised but were good enough for the purpose of demonstrating the usefulness of the suggested method. Since identifying mild lung function reduction is a very difficult task for the SPECT system in a clinical context, it is important that the parameter values used are optimised.

In the literature, a range of lung SPECT imaging parameter values are used [3,5-9] depending on the different tasks addressed. For example, optimal parameter values for qualitative analysis might not be optimal for quantitative analysis. To our knowledge, only Palmer et al. has reported an optimisation procedure and that is for qualitative analysis. Guidance in finding the optimal parameter values can be obtained using trade-off plots of image quality parameters such as contrast, noise and resolution [10-12] or using normalised mean square errors $[13,14]$, but none of these strategies are suitable for optimising the parameter values for the $\mathrm{CV}_{\mathrm{T}}$ method. The optimal parameter values have to be found in relation to the images used in the $C V_{T}$ method and its quantitative task. The images' visual appearance is therefore of secondary importance.

The ultimate test of the applicability of the $\mathrm{CV}_{\mathrm{T}}$ method will require access to a large group of well-defined healthy subjects and a large group of well-defined patients with mild COPD. Lacking access to these groups of subjects, Monte Carlo simulated lung SPECT images of software phantom lungs will be used.

The aim of this study was to determine, when using the $\mathrm{CV}_{\mathrm{T}}$ method, which parameter values of acquisition, reconstruction and analysis maximise the separation between the activity distributions in the lungs of healthy subjects and in the lungs of subjects with mild COPD (small heterogeneity variations).

\section{Methods}

The software lung phantom and Monte Carlo simulations The NCAT phantom [15] with a lung volume (air, blood and parenchyma) of 4.21 was used. The lung volume corresponded to a 65-year-old male $(70 \mathrm{~kg}, 180 \mathrm{~cm})$ in a supine position and in the middle of the respiratory cycle $[16,17]$. The arms were held over the head. The respiratory motion and heartbeat of the phantom were activated throughout the study, i.e. the phantom was dynamic. The phantom consisted of a $256 \times 256 \times 256$ matrix with a voxel size of $0.165 \times 0.165 \times 0.165 \mathrm{~cm}^{3}$. The muscle, fat, lung, spine bone, rib bone, blood and heart were the selected tissues. Densities and elemental compositions of these tissues were obtained from ICRP 89 [18] and XCOM photon interaction cross sections from Berger et al. [19].

Two different activity distributions were defined (Table 1). The activity distribution in the lungs of a healthy subject (hereafter called healthy distribution) was represented by a homogeneous distribution throughout the lungs. The activity distribution in the lungs of a subject with mild COPD (hereafter called COPD distribution) was represented by a non-homogeneous distribution modelled as small spherical lesions with a diameter of $1 \mathrm{~cm}$ within an otherwise homogenous activity distribution. The activity concentrations in the lesions were $50 \%$ of the concentration in the healthy surrounding lung tissue. The reduced activity levels in the lesions reflect the magnitude of the reduced ventilation or perfusion. No activity was located elsewhere in the body. The density of the lesions was the same as for the healthy lung simulating lack of activity beyond narrow and/ or closed airways. In Table 1, coronal slices are shown, including motion artefacts, to illustrate the distributions observed by the gamma camera during projection acquisition.

SPECT projections from the activity distributions were simulated using the SIMIND software, version 4.9d [20]. The projection data incorporated the effects of non-uniform attenuation and scatter. The isotope ${ }^{99 \mathrm{~m}} \mathrm{Tc}$ was used corresponding to the use of Technegas in a clinical setting. The energy window was set between 130 and $154 \mathrm{keV}$ to improve contrast-to-noise ratio [21,22]. A non-circular gamma

Table 1 Activity distributions and their descriptions

\begin{tabular}{ll}
\hline Coronal slice & $\begin{array}{l}\text { Distribution number and } \\
\text { description }\end{array}$ \\
\hline Healthy \\
distribution
\end{tabular}

Coronal slices include motion artefacts. 
camera rotation orbit was used, corresponding to the autocontouring system used in our clinic. The centre of rotation to collimator distance varied between 17 and $25 \mathrm{~cm}$.

Simulations were made for a GE Infinia gamma camera (Milwaukee, WI, USA) (0.95-cm-thick NaI crystal) equipped with a low-energy high-resolution collimator (LEHR) and a low-energy general-purpose collimator (LEGP). Projections were collected at 128 different angles, equally spaced, over $360^{\circ}$, in $128 \times 128$ matrices $\left(0.33 \times 0.33 \mathrm{~cm}^{2}\right.$ per detector element). A total of $1.8 \times 10^{10}$ photons were simulated with the homogeneous activity concentration, resulting in projections with low noise levels. The CV of one pixel element within the high count area of the lung in one single projection, determined from three consecutive simulations, was $0.5 \%$.

\section{Normalisation and statistical noise}

Clinically realistic noisy projections were generated by scaling the simulated projections and then replacing the pixel values by random deviates drawn from a Poisson distribution $[12,13]$. The total number of counts in the simulated projections for both activity distributions was set according to Table 2 . These values correspond to activities of 25 and $125 \mathrm{MBq}$ contained in the lungs using LEGP and LEHR collimators, respectively [3]. For each activity distribution, 40 noise realisations were created, simulating 40 SPECT acquisitions of the same activity distribution.

\section{SPECT reconstruction and filtering}

Each set of 40 noisy projections was reconstructed using the iterative ordered subset expectation maximisation (OSEM) [23] reconstruction software developed at John Hopkins University, Baltimore, MD, USA. The reconstruction included correction for attenuation, scatter and geometrical collimator detector response (CDR) (also known as point spread function (PSF) reconstruction and resolution recovery). Since it is a simulation study, the phantom attenuation coefficients were known and attenuation correction was performed using the average attenuation coefficient map over the respiratory cycle of the phantom. The scatter correction was performed using effective source scatter estimation (ESSE) [13,24]. An analytic geometrical model for CDR compensation was used for each collimator. The reconstructions were performed using 16 subsets and ten different numbers of iterations (see Table 3). The isotropic voxel size in a reconstructed image

Table 2 The total number of counts in the 128 simulated projections

\begin{tabular}{lll}
\hline & \multicolumn{2}{l}{ Administered activity } \\
\cline { 2 - 3 } & $\mathbf{2 5} \mathbf{~ M B q}$ & $\mathbf{1 2 5} \mathbf{~ M B q}$ \\
\hline LEGP & $1.23 \times 10^{6}$ & $6.14 \times 10^{6}$ \\
LEHR & $0.73 \times 10^{6}$ & $3.64 \times 10^{6}$ \\
\hline
\end{tabular}

Table 3 Variables used in the study

\begin{tabular}{|c|c|}
\hline & Values \\
\hline \multicolumn{2}{|l|}{ Acquisition } \\
\hline Activity levels & 25 and $125 \mathrm{MBq}$ \\
\hline Collimators & LEHR and LEGP \\
\hline \multicolumn{2}{|l|}{ Reconstruction } \\
\hline Number of iterations & $\begin{array}{l}2,4,6, \ldots, 20 \text { with } 16 \text { subsets, i.e. } \\
32 \text { to } 320 \text { updates }\end{array}$ \\
\hline Cutoff frequencies & $0.4,0.5,0.6,0.7 \mathrm{~cm}^{-1}$ and no filtering \\
\hline \multicolumn{2}{|l|}{ Analysis } \\
\hline Kernel edge lengths & $\begin{array}{l}1.0,1.7,2.3 \text { and } 3.0 \mathrm{~cm} \text { (i.e. } 3,5,7 \\
\text { and } 9 \text { voxels) }\end{array}$ \\
\hline Volume of analysis & The whole lung and the reduced lung \\
\hline
\end{tabular}

was $0.33 \times 0.33 \times 0.33 \mathrm{~cm}^{3}$. The reconstructed images were post-filtered with a Butterworth filter [25] with four different cutoff frequencies and a power of 6 (Equation 1) or not filtered at all.

$$
A(f)=\left\lfloor 1+\left(\frac{|f|}{Q}\right)^{p}\right\rfloor^{-0.5}
$$

where $A(f)$ is the amplitude of the filter at a spatial frequency $f$ and $Q$ is the cutoff frequency that controls rolloff and $p$ is the power factor.

\section{Analysis}

The $\mathrm{CV}_{\mathrm{T}}$ method used for the evaluation generates a global measure of inhomogeneity, the $\mathrm{AUC}\left(\mathrm{CV}_{\mathrm{T}}\right)$-value, constructed using a kernel-based CV distribution [3,4]. Four kernel edge lengths were used in the evaluation (see Table 3). Each combination of activity level, collimator, number of updates, cutoff frequency of the noise reduction filter and kernel edge length is here called a design. The total number of designs was 800 . For each design and activity distribution (Table 1), 40 density curves of CV values based on the 40 noise realisations were generated. For each design, the density curves of the healthy distribution generated a mean frequency function. Its modal value was then used as the threshold $\mathrm{CV}_{\mathrm{T}}$ value. Finally, the AUC for $\mathrm{CV}$ values greater than $C_{\mathrm{T}}$ (i.e. $\mathrm{AUC}\left(\mathrm{CV}_{\mathrm{T}}\right)$ ) was calculated for the two activity distributions. The same procedure was repeated for each design.

\section{Statistic evaluation}

For each design and activity distribution (Table 1), 40 AUC $\left(\mathrm{CV}_{\mathrm{T}}\right)$ values were obtained from the 40 density curves (described above). The non-parametric Mann-Whitney $U$ test was used when comparing results from the two activity distributions (healthy and COPD cases). Since the number of values in each group is 40 , i.e. larger than 10, the normal approximation to the Mann-Whitney $U$ distribution can 
be applied and $Z$-statistics and $p$ values can be calculated [26]. The Bonferroni correction [27] for multiple comparisons was applied reducing the rejection level of the statistical hypothesis test by a factor of $1 / 800$. In this study, the $p$ values were not only used for hypothesis testing but also to rank the designs. The $p$ values corresponding to the negative $Z$-statistic values were not included in the ranking. The lowest $p$ value corresponds to the best separation between the $\mathrm{AUC}\left(\mathrm{CV}_{\mathrm{T}}\right)$ values for the healthy and COPD distributions and defines the optimal design.

\section{Defining lung volumes}

The lung voxels in the homogenous distribution (Table 1), containing values greater than half the maximum lung value, were set to represent the segmented lung. A reduced lung volume was made by eroding the outer boundary of the segmented lung by one voxel. Both the whole lung and the reduced lung volumes were evaluated in the final optimisation (see Table 3).

\section{Image and computer processing}

The addition of Poisson noise, post-filtration, AUC $\left(\mathrm{CV}_{\mathrm{T}}\right)$ calculation and Mann-Whitney $U$ test was performed using in-house software developed in Interactive Data Language (IDL; ITT Visual Information Solutions, Boulder, CO, USA).

\section{Results}

When the whole lung is analysed, the lowest $p$ values result from using LEHR-125 MBq designs. LEHR-125 MBq designs placed in order of rank based on resulting $p$ values and thereafter grouped by kernel size show the lowest $p$ value for the smallest kernel size, i.e. with an edge length of $1.0 \mathrm{~cm}$ (see Figure 1b). When the designs are grouped by number of iterations, a minimum is found at four iterations, i.e. 64 updates (see Figure 1c). When grouped by cutoff frequency of the Butterworth filter, a minimum at 0.6 to $0.7 \mathrm{~cm}^{-1}$ is found (see Figure 1d). The lowest $p$ value for these designs is $p=4.1 \times 10^{-13}$. Designs generating the 10 lowest $p$ values are listed in Table 4 . The lowest $p$ value for a LEGP-125 MBq design is $p=8.0 \times 10^{-4}$, a LEGP$25 \mathrm{MBq}$ design $p=0.016$ and for a LEHR-25 MBq design $p=0.020$. Also shown in Figure 1 is that a LEHR-125 MBq design can result in a higher $p$ value than the best LEGP$125 \mathrm{MBq}$ design, e.g. if a kernel edge length of $3.0 \mathrm{~cm}, 288$ updates and a cutoff frequency of $0.4 \mathrm{~cm}^{-1}$ are chosen, $p=0.0026$ will result.

Also, for the reduced lung, the lowest $p$ values result from LEHR-125 MBq designs. Low $p$ values for LEHR$125 \mathrm{MBq}$ designs are found for the three largest kernel sizes with an edge length of 1.7 to $3.0 \mathrm{~cm}$, six to eight iterations (i.e. 96 to 128 updates) and a cutoff frequency of $0.7 \mathrm{~cm}^{-1}$ (see Figure 1). The lowest $p$ value for these designs is $p=2.8 \times 10^{-14}$. Designs generating the 10 lowest $p$ values are listed in Table 5 . The lowest $p$ value for a LEGP-125 MBq design is $p=2.0 \times 10^{-8}$, a LEGP$25 \mathrm{MBq}$ design $p=0.0043$ and for a LEHR-25 MBq design $p=5.2 \times 10^{-4}$. An example of a combination of a LEHR-125 MBq design for the reduced lung with a kernel edge length of $3.0 \mathrm{~cm}, 32$ updates and a cutoff frequency of $0.4 \mathrm{~cm}^{-1}$ results in a $p$ value as high as 0.26 .

Figure 2 shows the $\mathrm{CV}$ mean density curves and AUC $\left(\mathrm{CV}_{\mathrm{T}}\right)$ distributions of the healthy and the COPD distributions for the top-ranked LEHR-125 MBq design and a reduced lung. The threshold value $\left(\mathrm{CV}_{\mathrm{T}}\right)$ for this design is $23.0 \%$. The mean frequency function of the imaged COPD distribution is shifted towards higher $\mathrm{CV}$ values compared to the mean frequency function of the imaged healthy distribution (see Figure 2a). This shift results in higher AUC $\left(\mathrm{CV}_{\mathrm{T}}\right)$ values for the COPD distribution compared to the healthy distribution, as shown in Figure 2b,c. For this design, there is no overlap of the two $\mathrm{AUC}\left(\mathrm{CV}_{\mathrm{T}}\right)$ distributions, and therefore the resulting $p$ value is very low (see Table 5).

For the majority of the evaluated designs, the MannWhitney $U$ test results in positive $Z$-statistic values. A positive $Z$-statistic appears when the $\mathrm{AUC}\left(\mathrm{CV}_{\mathrm{T}}\right)$ distribution of the imaged COPD distribution appears to the right of the imaged healthy distribution, as in Figure $2 \mathrm{~b}$. A negative $Z$-statistic value, on the other hand, appears when their relative positions are reversed. Only positive $Z$-statistic values are generated for the LEHR-125 MBq designs as valid for both lung volumes. Negative $Z$-statistic values instead most frequently appear for the LEGP collimator, low numbers of updates, low cutoff frequencies of the filter and for designs including the whole lung.

\section{Discussion}

In the present study, we have optimised the $\mathrm{CV}_{\mathrm{T}}$ method with respect to the parameter values of acquisition, reconstruction and analysis in order to maximise the ability of the method to distinguish between images of healthy and mild COPD distributions. We have found that $125 \mathrm{MBq}$ and a LEHR collimator together with a Butterworth post-filter with a cutoff frequency of 0.6 to $0.7 \mathrm{~cm}^{-1}$ should be used. When the whole lung was analysed, 64 updates of the OSEM algorithm together with a kernel edge length of $1.0 \mathrm{~cm}$ gave the best results. For the reduced lung volume, a larger number of updates and larger kernel sizes were needed.

In our previous work [3], we evaluated the outcome of the $\mathrm{CV}_{\mathrm{T}}$ method using a variety of different simulated COPD activity distributions. The COPD distribution that was most difficult to separate from the simulated healthy distribution was chosen in this study. The aim was to find the parameter values that gave the $\mathrm{CV}_{\mathrm{T}}$ method the 


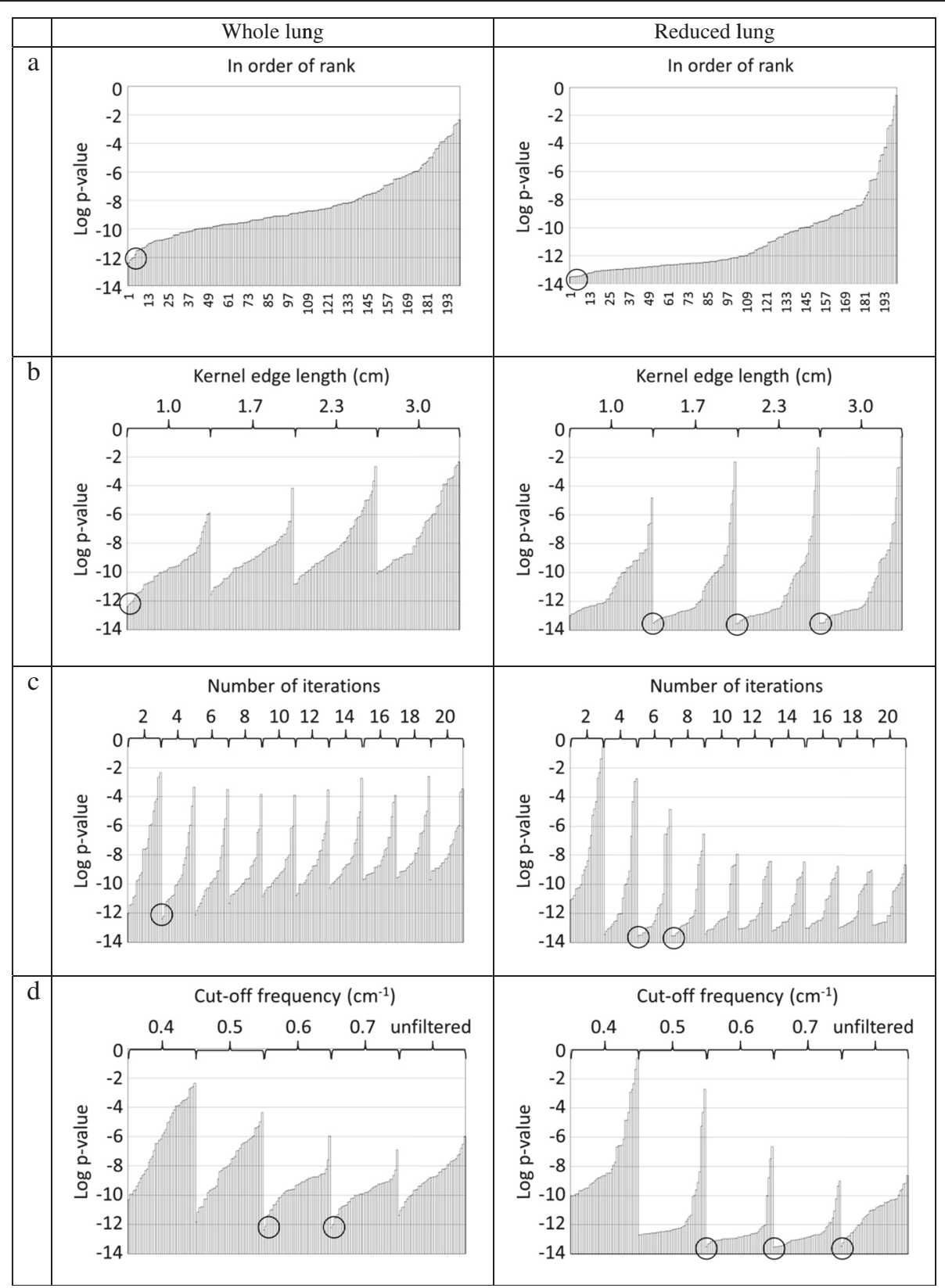

Figure $1 p$ values for all the LEHR-125 MBq designs. Designs for the whole lung are shown in the left column and for the reduced lung in the right column. In row (a) the designs are placed in order of rank based on resulting $p$ values. In row (b) the designs are grouped by kernel size, row (c) by iteration number and in row (d) by cutoff frequency of the Butterworth filter. The lowest $p$ values are encircled.

best chance of succeeding in identifying the smallest lesions. This non-homogeneous activity distribution was created with the aim of mimicking mild COPD where the reduced activity concentration in the small spherical lesions reflects the magnitude of reduced ventilation. The reduction of ventilation of $50 \%$ in $10 \%$ of the lung volume corresponds to a $5 \%$ total reduction of the functioning lung tissue. Compared to the normal range of spirometric variables of about $15 \%$ to $20 \%$, we believe this distribution would be very representative of a case of mild COPD. In this distribution, the lesions are evenly distributed throughout the whole lung volume. Grouped lesions have been shown [3] to be easier to resolve compared to evenly distributed lesions and were therefore not chosen in this study.

Since the spatial resolution of the SPECT system using an LEHR collimator is about $1.5 \mathrm{~cm}$ (expressed as FWHM at the applied orbit), the COPD activity distribution illustrates activity inhomogeneities that are blurred, resulting in reduced contrast. In the reconstructed images, most of the 
Table 4 The 10 designs generating the lowest $p$ values for the whole lung

\begin{tabular}{lllll}
\hline $\begin{array}{l}\text { Order } \\
\text { of rank }\end{array}$ & $\begin{array}{l}\text { Kernel edge } \\
\text { length } \mathbf{( c m )}\end{array}$ & $\begin{array}{l}\text { Number of } \\
\text { iterations } \\
\text { (updates) }\end{array}$ & $\begin{array}{l}\text { Cutoff } \\
\text { frequency } \\
\left(\mathbf{c m}^{-1}\right)\end{array}$ & $\boldsymbol{p}$ value \\
\hline 1 & 1.0 & $4(64)$ & 0.6 & $4.1 \times 10^{-13}$ \\
2 & 1.0 & $4(64)$ & 0.7 & $6.2 \times 10^{-13}$ \\
3 & 1.0 & $6(96)$ & 0.6 & $7.9 \times 10^{-13}$ \\
4 & 1.0 & $2(32)$ & 0.7 & $9.4 \times 10^{-13}$ \\
5 & 1.0 & $6(96)$ & 0.5 & $1.5 \times 10^{-12}$ \\
6 & 1.0 & $6(96)$ & 0.7 & $2.8 \times 10^{-12}$ \\
7 & 1.7 & $4(64)$ & 0.7 & $3.0 \times 10^{-12}$ \\
8 & 1.0 & $2(32)$ & 0.6 & $3.7 \times 10^{-12}$ \\
9 & 1.0 & $2(32)$ & Unfiltered & $4.2 \times 10^{-12}$ \\
10 & 1.0 & $8(128)$ & 0.6 & $4.5 \times 10^{-12}$ \\
\hline All & 1.0 &
\end{tabular}

All designs use LEHR collimator and $125 \mathrm{MBq}$.

inhomogeneities are due to an insufficient number of counts and few are due to true inhomogeneities. Despite these difficulties, a separation between the healthy and COPD distribution was found.

Tables 4 and 5 show good combinations of kernel sizes, number of updates and cutoff frequencies for LEHR-125 MBq designs. The differences between the 10 designs listed were small and all of them are good choices. The important message is that even for LEHR$125 \mathrm{MBq}$ designs, a bad combination of the parameter values would reduce the difference between a healthy

Table 5 The 10 designs generating the lowest $p$ values for the reduced lung

\begin{tabular}{|c|c|c|c|c|}
\hline $\begin{array}{l}\text { Order of } \\
\text { rank }\end{array}$ & $\begin{array}{l}\text { Kernel edge } \\
\text { length }(\mathrm{cm})\end{array}$ & $\begin{array}{l}\text { No of iterations } \\
\text { (updates) }\end{array}$ & $\begin{array}{l}\text { Cutoff frequency } \\
\left(\mathrm{cm}^{-1}\right)\end{array}$ & $p$ value \\
\hline 1 & 2.3 & $6(96)$ & 0.7 & ${ }_{-14}^{2.8 \times 10}$ \\
\hline 2 & 1.7 & $8(128)$ & 0.7 & ${ }_{-14}^{3.1} \times 10$ \\
\hline 3 & 2.3 & $8(128)$ & 0.6 & ${ }_{-14}^{3.1} \times 10$ \\
\hline 4 & 3.0 & $8(128)$ & 0.7 & ${ }_{-14}^{3.1 \times 10}$ \\
\hline 5 & 3.0 & $6(96)$ & 0.7 & $\begin{array}{l}3.3 \times 10 \\
-14\end{array}$ \\
\hline 6 & 3.0 & $6(96)$ & Unfiltered & $\underset{-14}{3.3} \times 10$ \\
\hline 7 & 3.0 & $4(64)$ & 0.7 & ${ }_{-14}^{3.5} \times 10$ \\
\hline 8 & 1.7 & $10(160)$ & 0.7 & ${ }_{-14}^{3.8 \times 10}$ \\
\hline 9 & 2.3 & $8(128)$ & 0.7 & ${ }_{-14}^{4.4} \times 10$ \\
\hline 10 & 1.7 & $6(96)$ & 0.7 & ${ }_{-14}^{4.7} \times 10$ \\
\hline
\end{tabular}

All designs use LEHR collimator and $125 \mathrm{MBq}$. and a diseased lung (Figure 1). All values have to be properly selected. One wrongly chosen parameter value can worsen the ability of the resulting system dramatically. An example, for the reduced lung, is LEHR$125 \mathrm{MBq}$ together with a kernel edge length of $3.0 \mathrm{~cm}$ and 64 updates. Changing the cutoff frequency from 0.7 to $0.4 \mathrm{~cm}^{-1}$ increased the $p$ value from $3.5 \times 10^{-14}$ to 0.0019 .

Using the optimal parameter values obtained in this work improved the ability of the $\mathrm{CV}_{\mathrm{T}}$ method to distinguish between mild COPD and healthy distributions compared to previously published results [3]. The optimisation reduced the resulting $p$ value from $5.8 \times 10^{-13}$ to $2.8 \times 10^{-14}$ for the COPD distribution and the reduced lung volume.

For quantitative assessment of small and less distinct lesions, as in this study, the image needed a high-count density and a high spatial resolution. The LEGP collimator could not resolve the small lesions sufficiently for either of the two activity levels. The study also showed that $25 \mathrm{MBq}$ together with the LEHR collimator had an excessively low count level. Increasing the number of counts increased the separation between the imaged healthy and COPD distributions. Therefore, in a clinical setting, it is important to reach a higher level of inhaled activity, which may be facilitated by coaching in breathing. To increase the separation further than shown in this study, even higher count levels than $3.64 \times 10^{6}$ are needed, which can be achieved by increasing the acquisition time. Our protocol (128 projections with a frame length of $10 \mathrm{~s}$ ) uses a total acquisition time of $11 \mathrm{~min}$ on a double-headed gamma camera. The effective dose was estimated to be $3.9 \mathrm{mSv}(1.9 \mathrm{mSv}$ for $125 \mathrm{MBq}$ of Technegas [28] and $2 \mathrm{mSv}$ for $\mathrm{CT}$ ).

Generally, 64 and 96 updates were found optimal for LEHR-125 MBq designs, which is consistent with our earlier result [12]. An even higher number of updates would increase the contrast recovery, but at the same time increase the noise level. An excessively high noise level seemed to diminish the advantage of increased contrast. A soft noise reduction filter with a cutoff frequency of 0.6 to $0.7 \mathrm{~cm}^{-1}$ was found optimal.

In the literature on quantitative methods [3,6,7], only use of a high-resolution collimator was found to agree with our results, while the count level and number of iterations varied radically. For qualitative assessment of pulmonary embolism, Palmer et al. [8] showed that a high-resolution collimator, a high number of total counts $\left(5.5 \times 10^{6}\right)$ together with a $128 \times 128$ matrix of the projections identified the highest number of lesion inserts in a thorax phantom. This agrees well with our results. On the other hand, they used only 16 updates. The contrast recovery achieved with as few as 16 updates would not recover the information that we are interested in. 

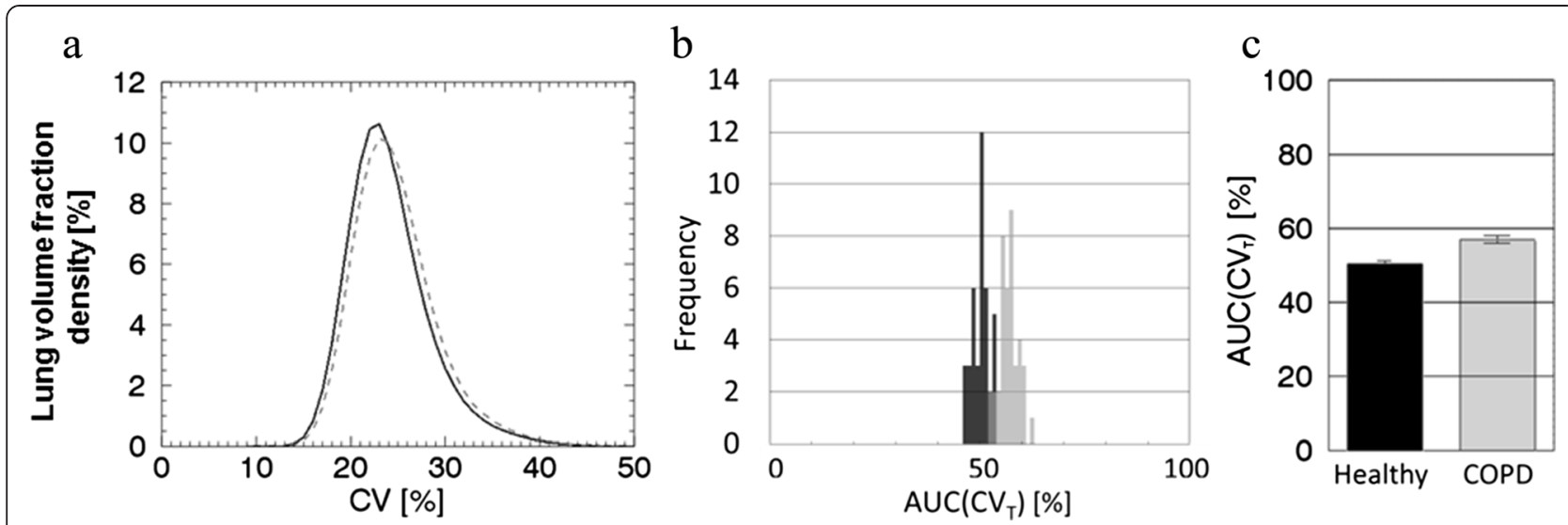

Figure 2 Mean density curves and $\mathbf{A U C}\left(\mathrm{CV}_{\mathrm{T}}\right)$ distributions. (a) The mean density curves of the 40 noise realisations of the $\mathrm{CV}$ values for the imaged healthy distribution (black line) and imaged COPD activity distribution (grey line) for the top-ranked LEHR-125 MBq design in Table 5 (a kernel edge length of $2.3 \mathrm{~cm}$, six iterations (96 updates) and a cutoff frequency of $0.7 \mathrm{~cm}^{-1}$ ). (b) Histograms of the AUC(CV $\mathrm{V}_{T}$ ) values of the 40 noise realisations for each activity distribution. (c) The same information as in (b) but visualised as mean values with $95 \%$ confidence intervals. Data are for the reduced lung.

It was better to use a large kernel size when the lung edge was excluded, but a small kernel size was slightly better when the whole lung was analysed. This can be explained by the following. In a static phantom, the diameter of the lesions was $1 \mathrm{~cm}$ and their depth was $50 \%$ of the surrounding healthy activity concentration. When the phantom incorporates respiratory motion, the lesions are smoothed, i.e. the lesions become broader and shallower. To this we add the limited resolution of the SPECT system which smoothed the lesions even further. When the lung edge was excluded from the imaged lung, a large kernel encloses more of the lesion gradient, compared to a small kernel, which resulted in higher CV values, i.e. it generated a greater separation between the healthy and COPD distributions. On the other hand, if the edge was included, large kernels at the edges will contain gradients due to lesions and lung edge. The lung edge is steeper and equal for both the healthy and COPD distributions while the lesions have shallower gradients. Therefore, a large volume at the periphery of the lung will not contribute to separating the healthy and COPD distributions. The remaining central volume seemed to be too small for the large kernel to gain many high $\mathrm{CV}$ values. Therefore, the resulting separation of the imaged distributions will not be greater than for a small kernel. In the case of a small kernel, the remaining central volume will just be somewhat smaller than the reduced lung and therefore only a small increase in separation between the healthy and COPD distributions was found when the lung edge was excluded.

Due to the limited spatial resolution of the SPECT system, the reconstructed activity distribution is blurred which is most clearly seen at the edge of the lung. The voxels involved in the lung edge will therefore result in high $C V$ values, which are not necessarily correlated to the inhomogeneity of the lung activity distribution. Therefore, a reduced lung volume was created with most of this edge effect excluded. Exclusion of the edge layer removes $21 \%$ of the original lung volume. This exclusion will be a disadvantage when the peripheral sub-pleural part of the lung is affected.

\section{Conclusions}

The best separation between a simulated healthy and a simulated mild COPD activity distribution using the $\mathrm{CV}_{\mathrm{T}}$ method was achieved using a total of at least $3.64 \times 10^{6}$ counts in the projections and by employing an LEHR collimator together with a Butterworth power 6 low-pass filter with a cutoff frequency of 0.6 to $0.7 \mathrm{~cm}^{-1}$. In the $\mathrm{CV}_{\mathrm{T}}$ method a kernel approach was used. When the whole lung was analysed, 64 reconstruction updates and a kernel edge length of $1.0 \mathrm{~cm}$ gave the best result. For the reduced lung volume, a greater number of updates and a larger kernel size are needed. Suboptimal selections of either cutoff frequency, number of updates and kernel size will reduce the imaging system's ability to detect mild COPD in the lung phantom, even for $3.64 \times 10^{6}$ counts together with an LEHR collimator.

\section{Abbreviations}

AUC: area under the curve; $A \cup C\left(C V_{T}\right)$ : area under the curve for $C V$ values greater than $\mathrm{CV}_{\mathrm{T}}$; CDR: collimator-detector response; COPD: chronic obstructive pulmonary disease; CV: coefficient of variation; $\mathrm{CV}_{\mathrm{T}}$ : CV threshold value; ESSE: effective source scatter estimation; SPECT: single-photon emission computed tomography.

\section{Competing interests}

The authors declare that they have no competing interests. 


\section{Authors' contributions}

PN participated in study planning, performed all lung SPECT analysis, evaluated the data and wrote the manuscript. AO participated in study planning and in writing. GAC participated in writing. MS and AG planned the study, participated in writing and provided grant funding. All authors read and approved the final manuscript.

\section{Acknowledgements}

This work was conducted at the Center for Medical Image Science and Visualization (CMIV) at Linköping University, Sweden. CMIV is acknowledged for its financial support and access to its research infrastructure. The reconstruction software developed at John Hopkins University, Baltimore, USA, was used in this work. The required scatter kernel files for the ESSE model were generated by Michael Ljungberg, Lund University, Sweden. Financial support was provided by the County Council of Östergötland, Sweden (ALF 2012-2013; grants to AG and MS) and the Medical Research Council of Southeast Sweden (FORSS 2012; grant to AG). Karl Wahlin, statistical advisor at the University of Linköping Sweden, is acknowledged for competent support. None of the authors have any conflicts of interest with regard to this work.

\section{Author details}

${ }^{1}$ Medical Radiation Physics, Department of Medical and Health Sciences, Linköping University, Linköping 581 83, Sweden. ${ }^{2}$ Center for Medical Image Science and Visualization (CMIV), Linköping University, Linköping 58183 , Sweden. ${ }^{3}$ Clinical Physiology, Department of Medical and Health Sciences, Linköping University, Linköping 581 83, Sweden. ${ }^{4}$ Department of Medical Physics, Karolinska University Hospital, Huddinge, Stockholm 141 86, Sweden.

Received: 30 September 2014 Accepted: 16 February 2015 Published online: 22 March 2015

\section{References}

1. Brown R, Woolcock AJ, Vincent NJ, Macklem PT. Physiological effects of experimental airway obstruction with beads. J Appl Physiol. 1969:27:328-35.

2. King GG, Harris B, Mahadev S. V/Q SPECT: utility for investigation of pulmonary physiology. Semin Nucl Med. 2010;40:467-73.

3. Norberg P, Persson HL, Carlsson GA, Bake B, Kentson M, Sandborg M, et al. Quantitative lung SPECT applied on simulated early COPD and humans with advanced COPD. EJNMMI Res. 2013:3:28.

4. Norberg P, Persson H, Schmekel B, Carlsson G, Wahlin K, Sandborg M, et al Does quantitative lung SPECT detect lung abnormalities earlier than lung function tests? Results of a pilot study. EJNMMI Res. 2014;4:39.

5. Bajc M, Neilly JB, Miniati M, Schuemichen C, Meignan M, Jonson B. EANM guidelines for ventilation/perfusion scintigraphy: part 1. Pulmonary imaging with ventilation/perfusion single photon emission tomography. Eur J Nucl Med Mol Imaging. 2009;36:1356-70

6. Eberl S, Chan HK, Daviskas E. SPECT imaging for radioaerosol deposition and clearance studies. J Aerosol Med. 2006:19:8-20

7. Nagao M, Murase K, Ichiki T, Sakai S, Yasuhara Y, Ikezoe J. Quantitative analysis of technegas SPECT: evaluation of regional severity of emphysema. J Nucl Med. 2000;41:590-5.

8. Palmer J, Bitzen U, Jonson B, Bajc M. Comprehensive ventilation/perfusion SPECT. J Nucl Med. 2001;42:1288-94.

9. Roach PJ, Schembri GP, Bailey DL. V/Q scanning using SPECT and SPECT/CT. J Nucl Med. 2013;54:1588-96.

10. Olsson A, Arlig A, Carlsson GA, Gustafsson A. Evaluation of reconstruction techniques in regional cerebral blood flow SPECT using trade-off plots: a Monte Carlo study. Nucl Med Commun. 2007;28:719-25.

11. Turkington TG, Wilson JM, Bowsher JE, Gilland DR. Limiting iterations vs. post smoothing for noise control in PET. In IEEE Nuclear Science Symposium Conference Record 4 2772-5, vol. Record. 2007:4:2772-5.

12. Norberg P, Bake B, Jacobsson L, Carlsson GA, Gustafsson A. Evaluation of reconstruction techniques for lung single photon emission tomography: a Monte Carlo study. Nucl Med Commun. 2007;28:929-36.

13. Larsson A, Mo SJ, Ljungberg M, Riklund K. Dopamine D2 receptor SPECT with (123)I-IBZM: evaluation of collimator and post-filtering when using model-based compensation-a Monte Carlo study. Phys Med Biol. 2010;55:1971-88
14. Beekman FJ, Slijpen ET, Niessen WJ. Selection of task-dependent diffusion filters for the post-processing of SPECT images. Phys Med Biol. 1998;43:1713-30

15. Segars WP, Tsui BMW. Study of the efficacy of respiratory gating in myocardial SPECT using the new 4D NCAT phantom. In: Nuclear Science Symposium Conference Record, 2001 IEEE; 4-10 November 2001. 2001. p. 1536-9. vol.1533.

16. Quanjer PH, Tammeling GJ, Cotes JE, Pedersen OF, Peslin R, Yernault JC. Lung volumes and forced ventilatory flows. Report working party standardization of lung function tests, European Community for Steel and Coal. Official statement of the European Respiratory Society. Eur Respir J Suppl. 1993;16:5-40.

17. Ibanez J, Raurich JM. Normal values of functional residual capacity in the sitting and supine positions. Intensive Care Med. 1982;8:173-7.

18. ICRP. Basic anatomical and physiological data for use in radiological protection: reference values. In International Commission on Radiological Protection: ICRP Publication 89; 2003.

19. Berger MJ, Hubbell JH, Seltzer SM, Chang J, Coursey JS, Sukumar R, et al. XCOM: photon cross section database (version 1.5). Gaithersburg, MD: The National Institute of Standards and Technology (NIST); 2010.

20. Ljungberg M, Strand S-E. A Monte Carlo program for the simulation of scintillation camera characteristics. Comput Meth Prog Biomed. 1989;29:257-72.

21. Gustafsson A, Arlig A, Jacobsson L, Ljungberg M, Wikkelso C. Dual-window scatter correction and energy window setting in cerebral blood flow SPECT: a Monte Carlo study. Phys Med Biol. 2000:45:3431-40.

22. Gustafsson A. Evaluation of attenuation and scatter corrections in lung and brain SPECT, Compilation thesis. Department of Radiation Physics: Göteborg University and Chalmers University of Technology; 2001.

23. Hudson HM, Larkin RS. Accelerated image reconstruction using ordered subsets of projection data. IEEE Trans Med Imaging. 1994;13:601-9.

24. Frey EC, Tsui BMW. A new method for modelling the spatially-variant, object-dependant scatter response function in SPECT. In: IEEE Nuclear Science Symp 1996. 1996. p. 1082-6.

25. Ott RJ, Flower MA, Babich JW, Marsden PK. The physics of radioisotope imaging. In The physics of medical imaging. Edited by Webb S. London: IOP Publishing Ltd; 1988: 142:318. p 197

26. Wahlin K. Tillämpad statistik- en grundkurs. Stockholm: Bonnier utbildning 2011.

27. Feinstein AR. Multiple stochastic testing. In Principles of medical statistics. Chapman and Hall/CRC; 2001

28. ICRP. Radiation dose to patients from radiopharmaceuticals. In International Commission on Radiological Protection: ICRP Publication 80; 2000

\section{Submit your manuscript to a SpringerOpen ${ }^{\odot}$ journal and benefit from:}

- Convenient online submission

- Rigorous peer review

- Immediate publication on acceptance

- Open access: articles freely available online

- High visibility within the field

- Retaining the copyright to your article

Submit your next manuscript at $>$ springeropen.com 